\title{
Was denkt im Individuum?
}

\section{Kollektivfiguren bei Ludwik Fleck, Tadeusz Bilikiewicz und Ludwig Gumplowicz}

\author{
Eva Johach
}

What is thinking inside the individual? Figurations of Collectivity in Ludwik Fleck, Tadeusz Bilikiewicz, and Ludwig Gumplowicz

\begin{abstract}
„What thinks in man, is not he himself, but his social community." These words by the early sociologist Ludwig Gumplowicz (1838-1909) were quoted several times by Ludwik Fleck (1896-1961) and seem to be in complete agreement with his own theory of thought collectives. The assumption that even scientific ideas were not so much generated by the scientist as an autonomous individual but rather by and within the social environment was still considered provocative by Fleck in the 1930s. This article will explore the implications of this assumption by comparing Fleck with Gumplowicz as well as with Tadeusz Bilikiewicz (1901-1980), a psychiatrist, philosopher and historian of medicine working like Fleck in the cultural milieu of Lwów/Lemberg.
\end{abstract}

Keywords: sociology of knowledge, thought collective, historical epistemology, science and environment, "struggle of the races"

Schlüsse/wörter: Wissenssoziologie, Denkkollektiv, Historische Epistemologie, Wissenschaft und Umwelt, "Rassenkampf"

Der größte Irrtum der individualistischen Psychologie ist die Annahme: der Mensch denke. [...] [W] as im Menschen denkt, das ist gar nicht er, sondern seine soziale Gemeinschaft. (Fleck 1935: 63)

Wer mit Ludwik Fleck vertraut ist, kennt diesen Satz, der sich in Flecks 1935 erschienene Monographie über die Entstehung und Entwicklung einer wissenschaftlichen Tatsache so nahtlos einfügt, dass er fast von ihm selbst stammen könnte. Ja, er scheint seine Theorie der Denkkollektive geradezu in nuce zu enthalten. Tatsächlich stammt der Satz aber nicht von Fleck, sondern ist dem Werk eines heute weitgehend unbekannten polnisch-jüdischen Soziologen entnommen, dem Grundriss der Soziologie von Ludwig Gumplowicz (1838-1909). 1885 verfasst, erschien es zwei Jahre später als Gumplowiczs Buch mit dem Titel Der Rassenkampf (1883). Das lässt nichts 
Gutes ahnen - hat Fleck etwa unkritisch einen Autor zitiert, der die Soziologie auf eine rassentheoretische Grundlage stellte?

Es ist, um es vorwegzunehmen, nicht davon auszugehen, dass sich Fleck mit der Soziologie Gumplowicz' näher auseinandergesetzt hat. Wie vor ihm bereits Wilhelm Jerusalem (1924), nutzte Fleck seine griffigen Sätze vor allem dazu, seiner These vom dezidiert kollektiven Charakter des Denkens Nachdruck zu verleihen. Noch 1935 ging Fleck davon aus, dass die Soziologisierung des Denkens - und gerade des wissenschaftlichen Denkens - einen Tabubruch darstelle, etwa wenn er schreibt: „Eine Art abergläubischer Furcht verhindert, das Allerintimste menschlicher Persönlichkeit, das Denken, auch einem Kollektive zuzusprechen" (Fleck 1935: 60). Selbst ein geschätzter Kollege wie Wilhelm Jerusalem glaube noch „an die Möglichkeit, ,rein theoretisch zu denken' und ,gegebene Tatsachen rein objektiv zu konstatieren"“ (ebd.: 66). ${ }^{1}$

Fleck sah ganz offensichtlich das Festhalten am Konzept des autonomen, denkenden Individuums als entscheidendes Hindernis an, um den Prozessen der Genese und Stabilisierung wissenschaftlicher Auffassungen auf die Spur zu kommen. Und wie Sylwia Werner gezeigt hat, spricht der Gegenwind, auf den Fleck stieß, sehr dafür, dass diese Annahme zutreffend war (Werner 2011), wobei hervorzuheben ist, dass dieser Gegenwind vor allem aus dem Lager der Philosophie kam. Wenn Fleck in den 1930er Jahren den Soziologen Gumplowicz durch mehrfaches Zitieren zum Gewährsmann für seine These von der sozialen Bedingtheit des Denkens erhob, dann richtete sich diese Positionierung nicht zuletzt gegen Flecks Lemberger Kollegen, den Philosophen und Wissenschaftshistoriker Tadeusz Bilikiewicz (1901-1980), der parallel zu Fleck das Projekt einer Historischen Epistemologie verfolgte und dabei ebenfalls auf den Begriff des Denkstils rekurrierte. Neben Wilhelm Jerusalem lieferte gerade dieser für Fleck eine Bestätigung, dass die Illusion des denkenden Individuums noch immer nicht überwunden sei. Bilikiewicz seinerseits verstand jedoch sehr genau, welche provokanten Konsequenzen mit einer Anwendung der Fleck'schen Denkstillehre auf den Prozess der Wissenschaften verbunden waren - bedeuteten sie doch nichts Geringeres als einen Angriff auf die Möglichkeit wissenschaftlicher Objektivität.

Bekanntlich hat Fleck seinen Ansatz in erster Linie für wissenschaftliche Denkkollektive ausformuliert; und nur an wenigen Stellen findet sich ein Hinweis darauf, dass er es für denkbar hielt, seine Denkstilllehre dafür zum Einsatz zu bringen, das „Denken eines Volkes, einer Klasse, einer wie immer gearteten Gruppe" vergleichend zu analysieren (Fleck 1935: 70). Anders als für Gumplowicz war die Kategorie der „Rasse“ generell keine Größe, die Fleck interessierte, ebenso wenig wie das Konzept der „Klasse“, das für Wissenssoziologen wie Mannheim eine zentrale Rolle spielte. Beide Kategorien hat Fleck recht pauschal von sich gewiesen. Seine Denkstillehre unterscheide sich 
grundlegend von sämtlichen Versuchen, die „Tatsache der soziologischen, gemeinschaftlichen Natur des Erkennens“ zur Grundlage zu nehmen, um entweder einen „National-“ oder „Rassengeist“ zu postulieren oder aber, politisch gegenläufig, „die politische Parole eines sozialen, klassenbedingten Wissens" zu untermauern (Fleck 1939a: 329).

Die durch das stichwortgebende Zitat von Gumplowicz aufgeworfene Frage „Was denkt im Individuum?" führt in eine Gemengelage von Kollektivfiguren, die für die Verankerung einer Soziologisierung des Denkens in Betracht kommen. Waren im Verlauf des 19. Jahrhunderts zahlreiche Versuche unternommen worden, Volk, Nation, Klasse, Gruppe oder andere Formen sozialer Gemeinschaft als Einheiten zu bestimmen, die das Denken von Individuen bestimmen, so fand seit den 1920er Jahren der Begriff des Denkstils immer häufiger in Verbindung mit dem Rassenbegriff Einsatz (Berg 2011). Unter Rückgriff auf die Völkerpsychologie kam es zu einer regelrechten „antisemitische[n] Vereinnahmung der Wissenssoziologie und des DenkstilsBegriffs" (Berg 2011: 99) - eine Tatsache, mit der Fleck Mitte der 1930er Jahre auch direkt konfrontiert wurde. ${ }^{2}$ Die höchst unterschiedlichen Implikationen, die mit der von Gumplowicz bezogenen These verbunden sein können, mögen Anlass genug sein, Flecks eigene Auffassung von der kollektiven Bedingtheit des Denkens schärfer zu konturieren. Ein geeigneter Ansatzpunkt hierfür ist eine genauere Bestimmung des Verhältnisses zwischen Individuum und sozialer Umwelt - ein Thema, das sowohl in der Soziologie Gumplowiczs als auch in der Auseinandersetzung zwischen Fleck und seinem Kollegen Bilikiewicz eine zentrale Rolle spielte. Ziel der hier vorgenommenen doppelten Konstellation ist letztlich, näher zu präzisieren, welche eigenen Akzente Fleck setzte, um der kollektiven Genese von Ideen und wissenschaftlichen Theorien eine produktive Seite abzugewinnen.

\section{Ludwig Gumplowicz: Vom Rassenkampf (1883) zum Grundriss der Soziologie (1885)}

Das Projekt einer Soziologisierung und Entindividualisierung des Denkens oder, allgemeiner formuliert: die Frage nach den kollektiven, sozialen Einflüssen, denen das Denken von Individuen unterliegt, wurde etwa seit den 1880er Jahren sowohl von der Ethnologie wie von der Soziologie verfolgt. Und es war gerade dieses Anliegen, das bereits im 19. Jahrhundert für einen Transfer völkerkundlicher Forschungen in die soziologische Theoriebildung sorgte. Während dieser Befund meist im Hinblick auf die Durkheim'sche (Religions-)Soziologie konstatiert wurde, lohnt auch eine Betrachtung der Genese der deutschsprachigen Soziologie, die im Spannungsfeld von Organizismus und Naturalismus darum bemüht war, den nicht-individuellen 
Charakter des Denkens unter Rückgriff auf die „Naturgesetze des Sozialen“ zu fundieren. Noch vor der Entwicklung einer eigenständigen Wissenssoziologie richtete sich dieser Versuch auch bereits auf Prozesse des Denkens und Erkennens (vgl. Durkheim/Mauss 1901/1902). In diese Früh- und Übergangsphase gehört auch Ludwig Gumplowicz. ${ }^{3}$

Als einer der Protagonisten in der Gründungsphase der deutschsprachigen Soziologie gehört Gumplowicz einer Fraktion an, die die neue Wissenschaft nicht, wie Dilthey, als Geisteswissenschaft verstehen, sondern sich an den Standards der empirischen Naturwissenschaften orientieren wollte. Das von ihm verfolgte Unterfangen einer ,Entsubjektivierung' des Denkens steht dabei im Kontext einer Überwindung idealistischer Prämissen, wie sie auch innerhalb der deutschen Völkerpsychologie, etwa bei Adolf Bastian, noch vorherrschend waren - jedoch ebenfalls zugunsten einer ,objektiven', naturwissenschaftlich orientierten Methode abgestreift werden sollten. Unter den deutschsprachigen Soziologen dieser Gründungsphase gehört Gumplowicz wohl zu denen, die sich am intensivsten mit der zeitgenössischen Völkerpsychologie und Ethnologie auseinandersetzten und deren Forschungsergebnisse auch für die Soziologie als wesentlich erachteten. Diese Position verbindet ihn mit Émile Durkheim, der Gumpowiczs Versuch einer Begründung der Soziologie zwar respektvoll zur Kenntnis nahm, die zeitgleichen Entwürfe seiner „organizistischen“ Kollegen allerdings vorzog (Anonym [Durkheim] 1885).

Im Zuge einer Suche nach elementaren „Naturgesetzen“ sozialer Prozesse werden typisch sozialdarwinistische Schlagworte wie der „Kampf“ zwischen den „Rassen“ und das Gesetz des Stärkeren zu maßgeblichen Kräften erhoben, die den Geschichtsverlauf im allgemeinen und die Entstehung des Staates im besonderen bestimmen. So schreibt Gumplowicz:

Jedes mächtigere ethnische oder soziale Element strebt danach, das in seinem Machtbereich befindliche oder dahin gelangende schwächere Element seinen Zwecken dienstbar zu machen. (Gumplowicz 1883: 161)

Zugleich aber legt Gumplowicz diesen Gesetzmäßigkeiten keine biologischen Kategorien zugrunde. Kampf und Krieg, Unterjochung und Ausbeutung, vor allem aber der Schlüsselbegriff der Assimilation sollen zu Leitbegriffen einer genuin soziologischen Wissenschaft werden. Was Gumplowicz als „Naturgesetz" des Sozialen versteht, ist freilich ein im Kern gewaltförmiger Prozess, in dem Assimilation eng mit Aneignung, Einverleibung und Untergang assoziiert ist. $^{4}$

Das entscheidende Moment, mit dem Gumplowicz den Übergang von einer allgemeinen Betrachtung der Menschheitsgeschichte hin zu genuiner Soziologie zu bewerkstelligen sucht, setzt ausgerechnet am Rassenbegriff an ein Umstand, der die Rezeption seiner Theorien nach 1945 nachhaltig behindert haben dürfte. Wie Gumplowicz immer wieder betont, stellt die 
Rasse keine biologische Einheit dar. Vielmehr handele es sich um „eine im Laufe der Geschichte, in und durch die sociale Entwicklung entstandene Einheit" (Gumplowicz 1905: 193). Anstatt „Rasse“ als eine essenzielle, vorgängige Größe zu betrachten, der Menschen qua Herkunft angehören, setzt Gumplowicz eine Soziologisierung des Rassenbegriffs ins Werk. Sein problematischer Versuch besteht darin, gerade über die Aneignung und soziologische Umwertung des Rassenbegriffs der Soziologie ein eigenes Forschungsfeld zu eröffnen. Die Homogenität von „Rassen“ wird auf „geistigen Austausch" zurückgeführt, durch den die Individuen einander ,assimiliert" werden und sich schließlich nicht nur in ihren Traditionen und Ansichten, sondern auch in ihrem physiognomischen Ausdruck angleichen. Unter Rückgriff auf einen lamarckistischen Rassenbegriff wird dabei selbst das gemeinsame „Blut“ zu einem nachträglichen Resultat aus Wirkungen, die man als soziale oder kulturelle Prozesse bezeichnen muss. Rassen entstehen nach Gumplowicz aus heterogenen Gruppen, die im Konflikt mit anderen heterogenen Gruppen stehen; im Geschichtsverlauf gehen aus diesen sozialen Gruppen - und dem in ihnen herrschenden Druck der Assimilation - erst die vermeintlich homogenen „Rassen“ hervor.

Diese Kämpfe im Hinblick auf die darin wirksamen und verallgemeinerungsfähigen „sozialen Gesetze“ zu ergründen, darin liegt für Gumplowicz der Übergang von einer (geschichtsphilosophisch fundierten) Völkerpsychologie in die Soziologie. Die Aufgabe der Soziologie bestehe, so lautet der Tenor beider Hauptwerke, in der Untersuchung der „Hervorbringung des Individuums durch die soziale Gruppe“. Und in diesem Kontext steht auch jenes Zitat, das Eingang in den Fleck'schen Text gefunden hat. Es findet sich in Gumplowiczs Grundriss der Soziologie und bildet den Auftakt des Teils über „Das Individuum und seine soziale Gruppe“, in dem er eine Umwelttheorie sozialer Gruppen entwickelt.

Der größte Irrtum der individualistischen Psychologie ist die Annahme: der Mensch denke. Aus diesem Irrtum ergibt sich dann das ewige Suchen der Quelle des Denkens im Individuum, und der Ursachen, warum es so und nicht anders denke, woran dann die Theologen und Philosophen Betrachtungen darüber knüpfen oder gar Ratschläge erteilen, wie der Mensch denken solle. Es ist das eine Kette von Irrtümern. Denn erstens, was im Menschen denkt, das ist gar nicht er sondern seine soziale Gemeinschaft, die Quelle seines Denkens liegt gar nicht in ihm, sondern in der sozialen Umwelt, in der er lebt, in der sozialen Atmosphäre, in der er atmet [...], und er kann nicht anders denken als so, wie es aus den in seinem Hirn sich konzentrierenden Einflüssen der ihn umgebenden sozialen Umwelt mit Notwendigkeit sich ergibt. (Gumplowicz 1905: 268)

Wie mehrfache Verweise auf Adolf Bastian zeigen, steht diese Positionierung im Kontext einer Auseinandersetzung mit der Völkerpsychologie, die für Gumplowicz eine wichtige Bedeutung für die Entwicklung der Soziologie besitzt und wie diese in den 1880er Jahren mit dem Problem einer Verabschiedung des „Individualismus“ kämpft. Obwohl der „Mensch“ 
weiterhin titelgebend für Bastians Wissenschaft bleibt (die er als „Wissenschaft vom Menschen" verstanden haben wollte), sieht Gumplowicz in ihr die Kerngedanken einer echten Soziologie keimhaft angelegt. Bastian habe eine folgerichtige Entwicklung hin zur Entindividualisierung beschritten, indem er sich bemühte, der Völkerpsychologie nicht mehr die alte idealistische, sondern eine neue, naturwissenschaftlichen Psychologie zugrunde zu legen: eine, die nicht mehr vom „Einzelmenschen“ ausgehe. So schrieb Bastian in seiner Vorgeschichte der Ethnologie:

Der Einzelmensch ist ein Unding, im besten Falle ein Idiot, nur in der Gesellschaft kommt der Gedanke durch Sprachaustausch zum Bewußtsein, die Menschennatur zur Geltung. Als das Primäre ergibt sich also der Gedanke der Gesellschaft, der Gesellschaftsgedanke, und erst aus ihm, durch spätere Analyse, wird der Gedanke des einzelnen zu gewinnen sein. (Zit. nach Gumplowicz 1905: 41)

Gumplowicz kommentiert: „Das sind goldene Worte, die wir als Motto der Soziologie akzeptieren“ (Gumplowicz 1905: 41). Mit diesen Formulierungen nämlich ist Bastian nahezu dort, wo sich auch Gumplowicz mit seiner Soziologie sieht: „In ihr verschwindet der Mensch; er kommt in erster Linie gar nicht in Betracht. Der sozialen Tatsache und Entwicklung erst folgt der Mensch und empfängt von ihr seinen Gedanken, seine Psyche“ (Gumplowicz 1905: 31). Aus den Individualgedanken sind Kollektivgedanken geworden, und der Einzelmensch ist jetzt etwas, was getrost der Philosophie überlassen werden kann.

Insgesamt kommt Gumplowicz in seiner Auseinandersetzung mit Bastian jedoch zu einem zweischneidigen Resultat: Einerseits habe er mit seinen Schriften Die Vorgeschichte der Ethnologie (1880), Der Völkergedanke im Aufbau einer Wissenschaft vom Menschen (1881), sowie den Allgemeinen Grundzügen der Ethnologie (1884) wichtige Grundlagen geschaffen, die für eine Soziologie seiner Diktion maßgeblich und unverzichtbar sind. Andererseits bescheinigt er Bastian, bei allen Anstrengungen einen ,unverdauten Rest von philosophischem Idealismus" beibehalten $\mathrm{zu}$ haben. Letztlich sei es, so Gumplowicz, die „Psyche“ selbst, die weiterhin das leitende Agens bleibt und so als epistemologisches Hindernis der Transformation von Bastians „ethnischer Soziologie" in eine wahre Soziologie im Wege steht. Bei aller Modernisierung der psychologischen Grundlagen sei Bastians Ansatz ein psychologischer, (noch) kein soziologischer.

Die intensive Auseinandersetzung mit der Völkerpsychologie ist damit ebenso sehr eine Verneigung vor deren Erkenntnissen wie auch eine Abgrenzung, die im klassischen Sinne als „boundary work“ (Gieryn 1983) zu verstehen ist. Dass Bastian $\mathrm{zu}$ soziologisch brauchbaren Ergebnissen gefunden hat, ist gewissermaßen der Tatsache geschuldet, dass er mit seiner Wissenschaft in etwa dieselbe Systemstelle zu belegen versucht, die Comte in seinem Stufenmodell der Wissenschaften der Soziologie zugesprochen habe. 
Ähnlich wie Comte der Soziologie, als der jüngsten der Wissenschaften, als der Wissenschaft der Zukunft, ihren in der Hierarchie der Wissenschaften einzunehmenden Platz (nach der Biologie) anweist, ähnlich beginnt Bastian seine Schrift zur Vorgeschichte der Ethnologie mit dem Hinweis auf die Stelle, welche die Ethnologie in der Entwicklung der Wissenschaften als deren jüngste einzunehmen hat. Schon dieses äußere Zusammentreffen zeigt, daß es sich hier vielleicht um ein und dasselbe Ding handelt. (Gumplowicz 1905: 39)

Diese Möglichkeit impliziert jedoch nicht nur Geistesverwandtschaft, sondern auch Konkurrenz. Sofern es darum geht, mithilfe der Soziologie zu den Naturgesetzen der Gesellschaft vorzudringen, muss dieses Unterfangen nicht nur gegenüber der Psychologie, sondern auch gegenüber der Völkerkunde verteidigt und deren Anspruch auf ebendiesen Gegenstand in die Schranken gewiesen werden.

Wie in Gumplowiczs Grundriss der Soziologie zum Ausdruck kommt, bildet die Menschheitsgeschichte als eine Geschichte von "Rassenkämpfen“ einen Rahmen, in dem auch allgemeinere Gesetze des Sozialen verhandelt werden können. Neben die Makroebene tritt eine Mesoebene, und der soziologische Blick richtet ins Innere der gesellschaftlichen Prozesse. Die soziale Gruppe tritt als das maßgebliche soziale Agens auf. Da sie zur kleinsten Einheit des Sozialen erhoben wird, erschließt sich von der Gruppe aus die entsubjektivierende Stoßrichtung von Gumplowiczs Soziologie. Wie ist vor diesem Hintergrund das Verhältnis von denkendem Individuum und den Kollektivgedanken zu bestimmen? An dieser Stelle kommt der Umwelt-Begriff ins Spiel. Die Umwelt ist es, von der jene entsubjektivierenden Effekte ausgehen, und sie ist auch das, worin sich (vermeintlich) individuelle Gedanken überhaupt erst bilden. Zu ihrer Charakterisierung greift Gumplowicz zu einer Kombination aus bakteriologischen und physikalischen Metaphern. Neben der Metaphorik der Ansteckung spricht Gumplowicz von „Strahlen“, die sich im Individuum wie in einem „Brennpunkt“ konzentrieren.

In der Mechanik und Optik kennen wir das Gesetz, wonach wir aus der Beschaffenheit des Einfallwinkels diejenige des Ausfallswinkels berechnen. Auf geistigem Gebiete existiert ein ähnliches Gesetz, nur können wir es nicht so genau beobachten. Aber jedem Einfallswinkel eines geistigen Strahles in unser Inneres entspricht genau ein gewisser Ausfallwinkel unserer Anschauung, unseres Gedankens, und diese unsere Anschauungen und Gedanken sind nur das notwendige Resultat der auf uns seit unserer Kindheit eindringenden Einflüsse. Dabei spielt das Individuum nur die Rolle des Prismas, das die Strahlen von außen empfängt und nachdem es dieselben nach festen Gesetzen gebrochen hat, wieder in einer bestimmten Richtung und in bestimmter Farbe durchläßt. (Gumplowicz 1905: 268 f.)

Diese Metaphorik erinnert an Gabriel Tarde, der in seinem zeitnah 1890 erschienenen Werk Les lois de limitation ebenfalls auf optische Brechungsmetaphorik zurückgriff und mit einer Metaphorik der Ansteckung verband. So spricht Tarde von „Nachahmungsstrahlen“, die den sozialen Raum durchqueren und sich in den Gehirnen der sozialen Individuen „brechen“ (Tarde 2009). 
Mit der Wahl dieser Metaphorik ist ein wesentlicher Effekt verbunden: die dezidierte Vermeidung der Frage danach, was sich in einem sozialen Individuum abspielt, zugunsten der Frage nach dem Input und Output. Gedanken sollen als soziale Phänomene studierbar werden, unabhängig von den Individuen, die sie denken. Der Griff zu den Metaphern der mechanischen Optik soll den unpersönlichen Charakter des Denkakts hervorheben, und stellt in einer fast schon absurd objektivistischen Adaption des naturwissenschaftlichen Blicks in Aussicht, die individuelle Rezeption und Variation kollektiver Gedanken beobachten, ihre Übertragungswege exakt verfolgen und gewissermaßen apparativ messen zu können.

Das Individuum ist in diesem Modell rezeptiv im physikalischen wie im biologischen Sinne: Zum einen ist es Akkumulator und Speichermedium früherer ,Eingaben', die seit frühester Kindheit an ihm vorgenommen wurden. Ja, es sind nicht nur die unmittelbar vorherrschenden Vorstellungen, sondern jahrtausendealte Prägungen, die sich im Individuum kondensieren. ${ }^{6}$ Zum andern ist das Individuum beziehungsweise sein Gehirn ein infektionsfähiges „Organ“ und als solches nicht nur zur mechanischen Reflexion, sondern auch zur Verarbeitung dieser Eingaben in der Lage - allerdings erneut in einem eher reproduktiven Sinne, da der Output durch die Umwelt geprägt ist. So schreibt Gumplowicz über die „Horden“ oder „Rudel“ gleichaltriger Kinder:

Ihre Anschauungen sind im großen und ganzen dieselben. Man impfte ihnen die gleichartige Bewunderung für gewisse Klassen von Dingen und Personen ein, gegen andere Dinge und Personen erfüllte man sie unwillkürlich mit demselben $\mathrm{Haß}$ und Abscheu, von dem man selbst beseelt war; ja bis auf den Geschmackssinn an Speise und Trank haben sie alle eine gleiche Richtung und Abrichtung empfangen - lauter Uhrwerke, die so gehen, wie man sie gerichtet und aufgezogen hat. Wer ist es nun, der hier denkt, fühlt, schmeckt - ist es das Individuum? Nein! Es ist die soziale Gruppe; es sind ihre Gedanken, ihre Gefühle, ihr Geschmack, ihre Anschauungen, also auch ihre Absichten und Zwecke, ihre Ziele und ihre Handlungen, deren neue Keime hier sich entwickeln; wie die Alten sungen, so zwitschern die Jungen. (Gumplowicz 1905: 270)

Die Umwelt tritt bei Gumplowicz also zum einen als formende Kraft auf, die auf die Organe des Denkens einwirkt und diese „infiziert“. Im Blick auf das Ganze des Kollektivs als dem zentralen Forschungsgegenstand einer wissenschaftlichen Soziologie hingegen erscheint die Umwelt weniger als biologischer Faktor denn als physikalischer Raum, der von Strahlen durchdrungen ist. Die im Kollektiv zirkulierenden Gedanken werden dabei in Analogie zu Lichtstrahlen gesetzt. In diesem zweiten Modell kommen die Individuen nicht mit ihren (infizierten) Denkorganen, sondern als (mechanische) Hindernisse in Betracht, als Prismen oder Linsen, an denen sich die (Gedanken-)Strahlen brechen und abgelenkt werden. Bei Gumplowicz erscheint unter diesen Bedingungen ein genuin eigenständiges Denken, ein autonomer kognitiver Akt als nahezu unmöglich - würde dies doch 
voraussetzen, dass sich ein Individuum von den Denkströmen seiner Umwelt völlig entkoppelt, isoliert oder ihnen gegenüber immunisiert. Allenfalls im Stadium des alten Mannes könne ein gewisser Grad eigenständigen Denkens erreicht werden. Gumplowicz rekurriert hier auf ein Narrativ, das für die Konzeption sozialer Evolution im 19. Jahrhundert generell kennzeichnend ist: das Fortschrittsnarrativ einer psychischen Entwicklung, die vom gänzlich kollektiv bestimmten Denken des primitiven Menschen zum eigenständig denkenden modernen Menschen führt. Entscheidend ist nun jedoch, dass Gumplowicz diese Entwicklung durchkreuzt und mit dem Rekurs auf Umwelt und Assimilation Faktoren einführt, die (im Regelfall) schon das Aufkommen individualisierter Denkakte unterbinden. Im Namen einer Soziologisierung des Denkens wird gleichsam eine sekundäre Form der Entindividualisierung postuliert, die unter dem Einfluss gesellschaftlicher Kräfte stattfindet: Das „Naturgesetz“ der sozialen Bewegung läuft bei Gumplowicz auf die Determination des individuellen Denkens durch den Druck der Anpassung und Assimilation hinaus.

Übrigens war es dieser Punkt, an dem Flecks Zeitgenosse Wilhelm Jerusalem die Position Gumplowiczs korrigiert und in einen wissenssoziologischen Ansatz weiterentwickelt hat. Im Verlauf der Kulturentwicklung trete nämlich eine zunehmende „Verselbständigung des Einzelmenschen“ ein, und ihre späte Frucht sei die Wissenschaft. Mit dieser entscheidenden Stufe der menschlichen Kultur kehre sich das Gumplowicz'sche Diktum um:

Was im Menschen denkt, wäre dann nicht, wie Gumplowicz behauptet, die soziale Gemeinschaft, sondern eben gerade er selbst, der sich von den Fesseln der sozialen Gebundenheit befreit hat. (Jerusalem 1924: 34)

Damit stellt Jerusalem das evolutionäre Fortschrittsnarrativ gleichsam wieder her - nicht ohne jedoch die These anzuschließen, dass auch die Denkgebilde moderner, „eigenkräftig“ gewordenen Menschen den Stempel kollektiver Vorstellungen tragen (Jerusalem 1924: 39 f.). Bei Gumplowicz schließt sich hingegen keine eigentliche Soziologie des Erkennens und auch keine vergleichende Soziologie von Wissensformen an. Sein Blick bleibt auf die geschichtliche Makroebene gerichtet, und dort, wo die soziale Binnenintegration in den Fokus rückt, wird der Aspekt der Reproduktion betont, während Innovation kaum möglich erscheint. Die Entwicklung einer Theorie interner Veränderung, ,a theory of internal change, i.e., changes occuring within a social system which are causa sui" (Horowitz 1980: 49), scheint Gumplowicz nicht interessiert zu haben. Momente der Abweichung oder auch nur Variation kommen in seinem Modell nicht in den Blick. Ein Grund hierfür könnte darin liegen, dass das Auftreten von Abweichungen von den dominanten Ansichten einer sozialen Gruppe - wie am Beispiel des zu autonomem Denken gelangten alten Mannes ersichtlich - an das Vorhandensein von Individualität gebunden bleibt; da die Individualisierung des 
Denkens jedoch im selben Atemzug zu einer Illusion und somit selbst zum Ausnahmefall erklärt wird, bleibt als Gesamteindruck der eines sozialen Prozesses, der von der Nivellierung aller Ansätze individueller Abweichung durch ein übermächtiges Kollektiv geprägt ist.

Gumplowicz ist bestrebt, das Individuum und die psychologische Binnenperspektive dadurch aus seinem Modell zu vertreiben, dass er es in einem umfassenden, nach ,physikalischen' Gesetzen funktionierenden sozialen System platziert. Das Individuum verschwindet unter dem Druck einer sozialen Umwelt, die einer Individualisierung des Denkens entgegenwirkt. Individuen geraten gewissermaßen in den Einflussbereich sozialer Kräfte, werden zu Kollektiven verbunden und in diesen einander assimiliert. Dass sich ihre Denkweisen einander angleichen und sie dabei ihren, individuellen' Charakter verlieren, ist Teil einer auf Assimilation basierenden „sozialen Bewegung“, die Gumplowicz soziologisch zu beschreiben versucht. Paradoxerweise ist es gerade der Rassenbegriff, mit dem Gumplowicz solche sozialen Dynamiken erfassen will - um damit eine vielfach für essenziell gehaltene Größe zum Ergebnis sozialer Interaktion und konflikthafter Auseinandersetzungen zu erklären. Die von ihm postulierte Naturgewalt wirkt stets auf eine Vereinheitlichung hin, und der Inbegriff dieser Vereinheitlichung ist die „Rasse“. Sie bildet gleichsam das Ergebnis sozialer Bewegung, die sich von außen betrachtet als Abfolge antagonistischer Kämpfe, von innen betrachtet als assimilatorische Angleichung von Individuen manifestiert. Dieser Rahmen, die Konzeption von Geschichte als Geschichte von Gruppen- und Rassenkonflikten, bewirkt nun aber auch, dass die Mikroperspektive auf die Ausformung konkreter Ideen bei Gumplowicz erst gar nicht in den Blick gerät. Im Gegensatz dazu steht nun der Ansatz Flecks, der sich gerade für die Mikroprozesse sozialen Erkennens interessiert. Wodurch sich sein Ansatz auszeichnet, ist insbesondere ein gänzlich veränderter Umweltbegriff, der sowohl soziologisch wie epistemologisch folgenreich ist.

\section{Wissenschaft unter Einfluss: Flecks Debatte mit Tadeusz Bilikiewicz}

Maßgeblich für Flecks Auseinandersetzung mit dem Umweltbegriff war allerdings nicht so sehr Gumplowicz, und auch die Umwelttheorie Uexkülls scheint für Fleck keine allzu große Rolle gespielt zu haben. Vielmehr war es Flecks Kollege, der Psychologe und Philosoph Tadeusz Bilikiewicz, mit dem Fleck einen intensiven Disput um den Umweltbegriff führte. In dieser Debatte, angesiedelt in der Wissenschaftskultur Lembergs, betonte Fleck in besonders deutlichen Worten die epistemische Produktivität von Umwelteinflüssen und schärfte, teils mit polemischer Verstärkung, seinen methodisch-theoretischen Zugang in mehrfacher Hinsicht. Geführt wurde die engagierte Debatte 
zwischen den beiden aus Lemberg stammenden Forschern in mehreren Nummern der Zeitschrift Preglad Wspótczesny [Zeitgenössische Rundschau]. Als konkreter Auslöser ging die gemeinsame Teilnahme beider an der Tagung der polnischen Ärzte und Naturforscher in Lemberg (1937) voraus, bei der Fleck ein kurzes Referat „Über spezifische Merkmale des serologischen Denkens“ und Bilikiewicz einen Vortrag mit dem Titel „Aus den Überlegungen über den „Sinn' der Geschichte“ hielt (Werner/Zittel 2011: 335). Während Fleck ein Thema wählte, das sich auf eine konkrete Denkstilveränderung innerhalb der Mikrobiologie bezog (und diese in erstaunlicher Kürze und Präzision auf anderthalb Seiten auf den Punkt brachte), ${ }^{7}$ deutet der von Bilikiewicz gewählte Vortragstitel bereits darauf hin, dass in dieser Debatte nichts Geringeres als das Ethos wissenschaftsgeschichtlicher Forschung auf dem Spiel stand. Die Schärfe, mit der Fleck auf Bilikiewiczs Forschungsansatz reagierte - vielleicht noch zusätzlich dadurch provoziert, dass dieser in seiner Rezension der betreffenden Tagung den Fleck'schen Vortrag gänzlich unerwähnt ließ -, dürfte sich auch daraus erklären, dass die Auseinandersetzung auf einem Gebiet geführt wurde, auf das sowohl Philosophie als auch Soziologie Anspruch erhoben: Historische Epistemologie. ${ }^{8}$

Flecks Debatte mit Bilikiewicz ist vor diesem Hintergrund $\mathrm{zu}$ sehen. Tadeusz Bilikiewicz lässt sich einer Forschungsrichtung zuordnen, die zu diesem Zeitpunkt bereits auf eine gewisse Tradition zurückblicken konnte: Gestützt auf einen Begriff des Denkstils, den Bilikiewicz von Heinrich Wölfflin und Karl Joël bezog, hatte er 1932 im Umfeld von Henry Sigrist mit einer wissenschaftsgeschichtlichen Arbeit zur Embryologie promoviert (Bilikiewicz 1932). Diese Fallstudie, in deren Mittelpunkt der so genannte „RokokoOvismus“ steht, wollte er als eine Fallstudie über die „komplizierte und geheimnisvolle Erscheinung des geistigen Kollektivlebens von einem völlig speziellen Standpunkte" verstanden wissen (zit. nach Fleck 1939a: 328). Im Begriff des Denkstils will Bilikiewicz vor allem epochenspezifische Denkstrukturen erfassen und in Analogie zu kunstgeschichtlichen Stilepochen bringen. Ein zentraler Befund seiner Studie über die Embryologie der Barockzeit bezieht sich entsprechend auf den konstitutiven Zusammenhang zwischen dem Erstarken des Individuums am Ende des Absolutismus und dem wissenschaftlichen Postulat vom Eigenleben der Spermien - flankiert von weiteren Analogien, die von einer zunehmenden gesellschaftlichen Autonomisierung des Individuums zeugen. Im Zentrum der Analyse steht also die Suche nach einheitlichen Denkmustern, die verschiedene Wissensfelder einer Zeit miteinander teilen und im Hinblick auf ihre formalen Eigenschaften von den Denkstilen anderer Epochen deutlich abgrenzbar sind. Obwohl beide Forscher auf den Stilbegriff rekurrieren, verbergen sich in diesem Begriff sehr unterschiedliche methodische Implikationen (Zittel 2007, 2011).

Der Konflikt zwischen den beiden Forschern entzündete sich jedoch nicht unmittelbar am Stilbegriff, sondern an einem anderen Punkt, der gleichwohl 
das unterschiedlich gelagerte Interesse an einer Geschichte der Wissenschaften deutlich werden lässt: an der Frage danach, welche Bedeutung der sozialen Umwelt im wissenschaftlichen Erkenntnisprozess zukommt. Zunächst gibt Bilikiewicz Fleck ohne weiteres zu, dass sich Umwelteinflüsse auch positiv auf den Forschungsprozess auswirken können - dies sei aber als Sonderfall zu betrachten: epistemologisch müsse die positive, heuristische Wirkung der Umwelt von den „schlechten“ Einflüssen abgetrennt werden. Das verstehend-rekonstruktive Anliegen des Historikers muss sich für Bilikiewicz mit dem normativen Anliegen des Epistemologen verbinden:

Der Historiker muß zwar anerkennen, daß die Annahme eines Einflusses der Umwelt auf die Wissenschaft in heuristischer Hinsicht sehr günstig ist, umso mehr muß der Epistemologe dafür sorgen, daß das Erkennen von solchen Einflüssen unabhängig wird (Bilikiewicz 1939a: 345).

Ganz im Einklang mit dieser Forderung verbindet Bilikiewicz seine Studie zur historischen Embryologie mit der Warnung an jeden Forscher, nicht dem „Herdentrieb“ zu verfallen (Bilikiewicz 1939b: 362). Historische Epistemologie, wie er sie begreift, besitzt damit eine normative Implikation für die Gegenwart. Anhand historischer Fallbeispiele galt es, Irrtümer von wahren Aussagen zu trennen - und sie als lehrreiches Zeugnis dafür heranzuziehen, wie schädlich sich der Einfluss der „Umwelt" auf den Forschungsprozess auswirkt. Fleck hingegen setzt den Akzent anders: Seiner Auffassung nach kann es nicht darum gehen, die „Denkzwänge“, unter denen wissenschaftliche Erkenntnisse historisch hervorgebracht wurden, nachträglich zu korrigieren. Die damit verbundene grundlegende Differenz kommt in folgender Entgegnung zum Ausdruck, in der Fleck seinen eigenen Ansatz - ausgehend von einem positivierten Umweltbegriff - gegenüber Bilikiewicz schärft:

Bilikiewicz behauptet, daß die Untersuchungen des Einflusses der Umwelt auf die
Wissenschaft darauf abzielen sollen, diese Einflüsse auf die Erkenntnisprozesse zu
beseitigen. Aber wie das? Die Ausgangssituation für jedes Erkennen ist gerade
durch die Umwelt, ihre Geschichte sowie deren aktuellen Status gegeben. Woher
sollen die Erkenntnisprozesse kommen, wenn keine neuen Situationen, keine
neuen Begriffe und keine neuen Fragen entstehen, die immer nur im Laufe eines
Gruppenlebens geboren werden? [...] Eine Sache ist der Herdentrieb, und etwas
anderes ist ein befruchtender, notwendiger Einfluß der Umgebung. Ein isolierter
Forscher bliebe ohne die auf ihn wirkenden Kräfte der Umwelt und ohne den
Einfluß der Evolution dieser Umwelt blind und gedankenlos. Den Einfluß der
Umgebung zu beseitigen bedeutet, die Erkenntnisprozesse mindestens zu hem-
men. (Fleck 1939b: 358) $\mathrm{Zu}$ historisieren ist aus Flecks Sicht also nicht erst die Erkenntnis, sondern bereits die Umwelt, in der sie stattfindet - und die in der historischen Rekonstruktion gerade in ihrer dynamischen Qualität wiedererscheinen soll. In diesem Sinne ist Flecks Einwand gegen Bilikiewicz zu verstehen, „daß es weniger wichtig ist, ganze Anschauungen und Theorien zu untersuchen", um dem Denkstil einer Epoche nahezukommen. Entscheidender sei die Analyse 
spezifischer "Stilfärbungen“ in den verwendeten Begriffen der jeweiligen Theorie (Fleck 1939a: 332). Fleck versucht gewissermaßen, auch in der Rekonstruktion nicht an den Ideen selbst, sondern an den kollektiven Dynamiken anzusetzen, die an der Hervorbringung wissenschaftlicher Tatsachen beteiligt sind. Dieses mikrosoziologische Anliegen ist bei Fleck - was seinen Ansatz für die gegenwärtige kulturwissenschaftliche Wissenschaftsforschung so anschlussfähig macht - mit einer semantischen Rekonstruktionsarbeit verbunden, mit einer besonderen Aufmerksamkeit für Metaphern und semantische Verschiebungen. Was nämlich, so Fleck, den Weg zum Denkstil einer Epoche am besten eröffnet, sei

die Aura der Begriffe, [...] ihre Stilfärbung, die sich im sprachlichen Gebrauch durch das Verwenden bestimmter Worte spiegelt, besonders wenn diese Worte metaphorisch verwendet werden. (Fleck 1939a: 332, Hervorhebung im Original)

Gegen Bilikiewicz versucht Fleck also seinen eigenen Denkstilbegriff so zu fokussieren, dass sich der Akzent von der Gesamtsicht auf ideengeschichtlich entstandene Theoriegebilde entfernt und sich stattdessen auf deren kollektives Zustandekommen selbst verschiebt.

Durch eine umfassende Positivierung der epistemologischen Bedeutung, die der kollektiven Umwelt eines Forschers zukommt, gelangt Fleck zudem zu einer Generalisierung der These von der kollektiven Bedingtheit des Denkens, die auch die Auffassung von Objektivität nicht unangetastet lässt. Der kollektive Charakter des Erkennens gewinnt bei ihm eine so generelle Qualität, dass er weder als Sonderfall, noch als eliminierbarer Einfluss betrachtet werden kann, der je zugunsten einer unverstellten Sicht auf den Gegenstand verlassen werden könnte. Das Kollektiv, das also, was für Fleck die Umwelt des Forschenden darstellt, hört auf, ein lediglich äußerer Einfluss zu sein und wird in die Erkenntnisbeziehung selbst integriert. Es wird zum Dritten zwischen Subjekt und Objekt erhoben und somit zu einem unhintergehbaren Tertium, das den Blick auf einen Gegenstand so unabdingbar formt, dass dieser eben stets nur "mit den Augen des Kollektivs" betrachtet, aufgefunden und interpretiert werden kann (Fleck 1935: 53, Fleck 1947: 411 f.). Die damit verbundenen weitreichenden Konsequenzen hat Bilikiewicz als Historischer Epistemologe klar erkannt; Flecks These, wonach es „unmöglich ist, ein allgemeines Wahrheitskriterium festzulegen“ und die Wahrheit stattdessen „nur als eine aktuelle Etappe eines Denkstils anerkannt wird“, habe eine entscheidende Konsequenz: Sie führe notwendig in einen „kognitive[n] Relativismus, der verschiedenen Bildern der Wirklichkeit, die aus unterschiedlichen Denkstilen erwuchsen, die gleiche Gültigkeit zugesteht - sogar dann, wenn diese Bilder widersprüchlich sind“ (Bilikiewicz 1939a: 342).

Die Fleck'sche Auffassung hat somit ernsthafte epistemologische Konsequenzen für eine Konzeption wissenschaftlicher Objektivität (Fagan 2009, Zittel 2011). Wenn nämlich der wissenschaftliche Erkenntnisprozess, wie 
Fleck betont, immer und notwendig durch einen kollektiven Denkstil konstituiert ist, dann fällt dieser Sichtweise ein Anliegen zum Opfer, das für die Historische Epistemologie Bilikiewiczs noch unabdingbar ist: auf eine wissenschaftliche Erkenntnis hinzuarbeiten, die frei von Einfluss ist. Die grundlegende Blickverschiebung, die mit Flecks Konzeption ins Spiel kommt, liegt darin, dass die Unterscheidung zwischen richtigen und falschen Erkenntnissen keine wissenssoziologisch interessante Frage mehr ist - ein Skandalon, das in der Debatte mit Bilikiewicz besonders deutlich hervortritt. Dass es möglich ist, ja möglich sein muss, die Erkenntnisprozesse von den verunreinigenden Einflüssen einer sozialen Umwelt zu befreien, war für Bilikiewicz eine unverrückbare epistemologische wie normative Grundlage, die wissenschaftshistorischen Studien zugleich Relevanz für die Gegenwart verleihen sollte. In der Rekonstruktion dürfen die Irrtümer der Wissenschaftsgeschichte nicht indifferent gegen die wahren Einsichten gestellt werden. Mit der besonderen Prägung des Fleck'schen Denkstilbegriffs ging diese Verankerung verloren. Nicht nur die verworfenen wissenschaftlichen Auffassungen der Vergangenheit, sondern jede wissenschaftliche Entdeckung ist für Fleck ein denkkollektiver Prozess, der in seinen Möglichkeiten durch den Denkstil begrenzt wird.

\section{Die Positivierung der sozialen Umwelt: Flecks Kollektive}

Die Debatte mit Bilikiewicz macht somit deutlich, dass der zwischen beiden ausgetragene Konflikt um die Methodik historischer Denkstilforschung erhebliche epistemologische Konsequenzen hat. Hiervon ausgehend ist abschließend nochmals auf Gumplowicz zurückzukommen - genauer: zur Frage, welche neuen Implikationen das von ihm entliehene Zitat in Flecks eigener Position gewinnt. Wie erwähnt, ist nicht davon auszugehen, dass Fleck sich näher mit den Texten von Gumplowicz auseinandergesetzt hat. Das von Gumplowicz bezogene Zitat muss vielmehr - ganz im Sinne der Fleck'schen Denkstillehre - als ein zirkulierendes Element betrachtet werden, das seine intendierten wie nicht-intendierten Wirkungen in der Fleck'schen Umwelt der 1930er Jahre entfaltet.

Dass Fleck gar nicht erst versuchte, Gumplowicz argumentativ gerecht zu werden, macht umso deutlicher, wie sehr sich Flecks Einsichten über das Entstehen von wissenschaftlichen Tatsachen auch auf sein eigenes Vorgehen anwenden lassen. Flecks eigener Einsatz des Zitats zeigt nämlich, dass die Gumplowicz'sche Auffassung gleichsam demselben Schicksal unterliegt, das Fleck zufolge alle Erkenntnisse ereilt:

sie wandern innerhalb der Gemeinschaft, werden geschliffen, umgeformt, verstärkt oder abgeschwächt, beeinflussen andere Erkenntnisse, Begriffsbildungen, Auffassungen und Denkgewohnheiten. (Fleck 1935: 58) 
Der Wert des Zitats liegt damit auf der funktionalen Ebene, in seinem Charakter als denkkollektive Intervention. Entwendet, entkontextualisiert, umgenutzt und polemisch eingesetzt, dient es Fleck dazu, ins Gebiet der Erkenntnistheorie vorzudringen, um dort mit dem ,Mythos' des erkennenden Subjekts aufzuräumen. Mit der „heiligen Scheu“, die viele seiner Kollegen (und zwar sowohl innerhalb der Philosophie als auch in der Soziologie) besitzen, soll möglichst gründlich Schluss gemacht werden. Zum andern aber ist der Einsatz des Zitates alles andere als eine Reproduktion, und die im Zitat versammelten Denkstilelemente erfahren einen deutlichen Färbungswechsel: Die „soziale Gemeinschaft", die Fleck im Sinn hat, stimmt mit der von Gumplowicz gezeichneten keineswegs mehr überein. Zwar gehen beide von der nicht hintergehbaren Einwirkung einer sozialen Umwelt auf das Denken aus. Allerdings gewinnt die Umwelt bei Fleck einen deutlich anderen Akzent. Zugespitzt gesagt, wird sie von einer ausschließlich hemmenden zu einer ermöglichenden Größe.

Dieser Färbungswechsel zeigt sich zunächst an der verschobenen Konnotation, die mit dem Wirken sozialer Umwelten verbunden wird. Gumplowicz hatte von einer „elementaren Naturgewalt" gesprochen, die eine Entstehung individueller Gedanken grundsätzlich verhindert und stattdessen eine wechselseitige Assimilation ins Werk setzt. Bei Fleck taucht eine ähnliche Formulierung auf, wenn er die soziale Gemeinschaft zu einem „Dritten“ macht und mit nicht hintergehbarer Macht ausstattet: „Es ist kreativ wie das Subjekt, widerspenstig wie das Objekt und gefährlich wie eine Elementargewalt" (Fleck 1960: 470). Dennoch war Fleck im seiner Umwertung des Kollektivbegriffs bestrebt, diese Elementargewalt nicht ausschließlich unter dem Gesichtspunkt ihrer negativen Wirkungen zu erfassen. Wer die „soziale Bedingtheit" des Denkens, so Fleck, „für eine leider existierende Unzulänglichkeit ansieht, die zu bekämpfen Pflicht ist, verkennt, daß ohne soziale Bedingtheit überhaupt kein Erkennen möglich“ ist (Fleck 1935: 59).

Ein weiterer entscheidender Färbungswechsel lässt sich dort feststellen, wo es um die "soziale Atmosphäre" geht, in der schon Gumplowicz jedes Denken angesiedelt hatte. Entscheidend ist hier, dass Fleck den Begriff der „Stimmung" sehr ernst nimmt und gerade deren veränderlichen Charakter für eine Flexibilisierung und Vervielfältigung der sozialen Einflüsse nutzbar macht. Von Fleck wird das Konzept der Stimmung für das eingesetzt, was Fleck als die „Beharrungstendenz der Meinungssysteme“ bezeichnet hat (Fleck 1935: 53), das heißt für das konservative Element in Denkstilen. Zugleich aber ist die „kollektive Erkenntnisstimmung“ (Fleck 1939a: 331) für Fleck der unabdingbare Ausgangspunkt für die Dynamik von Denkstilen. Fleck spricht etwa von jener „unruhige[n] Stimmung“, die einen Forscher erst dazu ermuntert, etwas Neues zu suchen und eine bestimmte, "gerichtete“ Denkbereitschaft auszubilden (ebd.: 330). In Bezug auf die von Bilikiewicz beschriebene Embryologie des Rokoko liege die entscheidende Aufgabe für die 
Wissenschaftsforschung darin, die Umwelt der Embryologen als eine zu einem gemeinsamen Denkstil führende gerichtete „kollektive Erkenntnisstimmung“ zu untersuchen.

Trotz seiner erkenntnisbegrenzenden Wirkungen ist und bleibt das Kollektiv für Fleck jedoch eine produktive Größe. Unter Rückgriff auf den Gestaltbegriff beschreibt Fleck dessen produktive Wirkungsmacht allerdings als einen prekären Mechanismus, bei dem Erkenntnis nicht grundsätzlich von Täuschung zu unterscheiden ist. Teil dieser epistemologischen Verschärfung ist, dass Fleck auch und gerade die naturwissenschaftliche Erkenntnis von einer „Harmonie der Täuschungen“ nicht ausnimmt. Gerade die experimentelle naturwissenschaftliche Forschung, Inbegriff objektiver Wissenschaft, wird in Flecks Darstellung zu einer Angelegenheit, die in massiver Weise anfällig für kollektive Täuschungen ist. Gerade hier, wo Wissenschafts- und Erkenntnistheoretiker ebenso wie mancher Wissenssoziologe zumindest noch die letzte Bastion der Objektivität vermuten würden, ist nach der Fleck'schen Auffassung nur noch ein Gestaltbildungsprozess zu beobachten, der mehr von der Eigentätigkeit sozialer, das heißt denkkollektiver Verstärkung Zeugnis gibt als von einer sukzessiven Annäherung an die objektive Wahrheit. Dass für Bilikiewicz als Philosoph die Auflösung von Objektivitätskriterien offenbar bedrohlicher war als für den Naturwissenschaftler Fleck, ist und bleibt vor diesem Hintergrund erstaunlich. Wenn man Flecks in dieser Hinsicht besonders instruktives Beispiel vom Versuch des Fleckfiebernachweises im Konzentrationslager Buchenwald bedenkt, bei dem ein Erreger „zweifelsfrei“ nachgewiesen wurde, obwohl er im zur Verfügung stehenden Serum gar nicht vorhanden war (Fleck 1946: 375-381), muss man immer wieder verblüfft sein, dass Fleck bereit war, die sich aus der sozialen Verstärkung ergebende „Harmonie der Täuschungen" nicht als existenzielle Bedrohung für die wissenschaftliche Forschung darzustellen, sondern als permanent gegebenen Mechanismus zu deuten. Sofern Objektivität nämlich auf die Realität schlechthin bezogen wird, kann es sie in dem Moment nicht mehr geben, wo die eine Realität durch eine Pluralität von Wirklichkeiten ersetzt wird. Dies bedeutet jedoch nicht, dass Fleck einen erkenntnistheoretischen Relativismus verfolgt (Löwy 1990, Borck 2004). Das besonders drastische Beispiel vom „Erregernachweis ohne Erreger“ ist hier aufschlussreich, denn es hat für die Fleck'sche Denkstillehre einen doppelten Status. Einerseits wird es von Fleck dazu benutzt, in besonders drastischer Weise den normalen soziologischen Hergang der Entstehung, Entwicklung und Stabilisierung von „Tatsachen“ zu demonstrieren und die komplexen Schritte einer solchen kollektiven Genese überhaupt erst sichtbar zu machen.

Jedoch: Die Pluralisierung der Kollektive und ihre wechselseitige Beeinflussung im ,interkollektiven Denkverkehr“ bietet zugleich die Gewähr dafür, dass es bei diesen temporär stabilisierten Denkgebilden nicht bleibt. „Die verwickelte Struktur der modernen Gesellschaft", so heißt es in Entstehung 
und Entwicklung einer wissenschaftlichen Tatsache, „,bringt es mit sich, daß Denkkollektive sich räumlich und zeitlich vielfach überkreuzen und in Beziehung zueinander treten" (Fleck 1935: 140 f.). Entscheidend für die positive Neukonturierung ist damit die Zirkulation, die von Fleck auf das Engste mit dem Konzept des Kollektivs verbunden wird. „Kollektivgedanke[n]", wie Fleck sie versteht, sind - ganz im Sinne von Gumplowicz einerseits Hervorbringungen der sozialen Gemeinschaft, die im Individuum denkt. Andererseits unterliegen diese jedoch einer permanenten Zirkulation, was eine Erstarrung von Kollektivgedanken stets aufs Neue verhindert; und da Fleck Kollektivwirkungen nach dem Vorbild eines informellen Gesprächs entwirft, spricht er jedem Individuum doch wieder Wirksamkeit zu. Jedes hinzukommende Individuum kann eine kollektive Stimmung verändern, und es ist an der Umwandlung von Denkstilen (unfreiwillig) dadurch beteiligt, dass es die im Kollektiv kreisenden Gedanken immer ein wenig anders versteht als die übrigen Mitglieder des Kollektivs und sie dadurch semantisch ablenkt (Fleck 1935: 60).

Die unabdingbare Verkopplung der kollektiven Genese und Stabilisierung von Erkenntnissen mit Prozessen der Zirkulation hat aber noch eine andere Konsequenz: Sie erlaubt es Fleck, den notwendig demokratischen Charakter von Denkkollektiven hervorzuheben - und damit eine epistemologische Bedingung indirekt $\mathrm{zu}$ einer politischen Forderung auszuweiten. Den Mechanismen der kollektiven Erkenntnisgewinnung wohne ein grundlegender Zug inne, den Cornelius Borck treffend Flecks "offene Epistemologie“ genannt hat (Borck 2004). Der naturwissenschaftliche Denkstil: das ist für Fleck nicht nur das Muster, an dem die kollektiv generierte und verstärkte Erkenntnis mit allen beschriebenen Konsequenzen demonstriert und als gleichsam notwendige „Harmonie der Täuschungen“ ausgewiesen wird. Darüber hinaus verbürgt der naturwissenschaftliche Denkstil einen Mechanismus, der von Fleck als „demokratisch“ bezeichnet wird: eine Erkenntnisstruktur, die eine dogmatische Schließung verhindert. Denkkollektive dürfen sich, wenn sie ihre epistemische Produktivität behalten und nicht zu Produzenten von dogmatischem Wissen werden wollen, nicht abschließen (vgl. Fleck 1935: 189).

\section{Fazit}

Im Zentrum der vorangegangenen Analysen stand die Frage nach den Implikationen von Flecks These, dass gerade das Denken als sozialer Prozess kat exochen gelten muss und es endlich an der Zeit sei, es aus der individualistischen Illusion zu befreien. Fleck hatte sich zur Verstärkung dieser Auffassung mehrfach auf den Soziologen Gumplowicz bezogen - in anderen 
Zusammenhängen aber deutlich gemacht, dass an die Stelle des denkenden Individuums keinesfalls die Rasse oder andere mysteriöse Größen rücken sollten. Während der erste Teil des Aufsatzes den Versuch Gumplowiczs rekonstruierte, gerade den Rassenbegriff zum Ausgangspunkt einer Soziologisierung des Denkens zu machen und darin kritisch an die Völkerpsychologie anzuschließen, widmete sich der zweite Teil der Debatte zwischen Fleck und Bilikiewicz Ende der 1930er Jahre. Näher beleuchtet wurde dabei das Verhältnis von Wissenschaft und Umwelt, aber auch die provokativen Konsequenzen des genuin Fleck'schen Denkstilkonzeptes, die sich in dieser Debatte besonders deutlich abzeichnen.

Was lässt sich nun ausgehend von der hier gewählten Konstellation über den von Fleck entworfenen Zusammenhang von Denken und Kollektiv folgern? Im Vergleich zu Gumplowicz zeigt sich zunächst, wie viel Wert Fleck darauf legte, den determinierenden Charakter der Umwelteinflüsse zu mindern, ja produktiv zu wenden. Für Gumplowicz stand die Umwelt in pessimistischer Diktion für eine soziale Totalität, die keinerlei Spielräume für individuelles Denken entstehen lässt. Bei Fleck hingegen wird diese Totalität pluralisiert; an die Stelle einer determinierend und vereinheitlichend wirkenden Umwelt tritt ein Ensemble sozialer Wirklichkeiten. In einem solchen Gefüge ist eine vollkommene Determination des Denkens nicht mehr möglich, und die soziale Umwelt verliert ihren übermächtigen Charakter. Zugleich geht aber auch ein fester Bezugspunkt für wissenschaftliche Objektivität verloren.

Flecks Akzentverschiebung im Denkstilbegriff steht in engem Zusammenhang mit einer Umwertung des Kollektivs, das bei Fleck von einer erkenntnishemmenden Größe zu einem Medium wird, in dem jede Erkenntnis gewonnen und ausgeformt wird. Wie deutlich wurde (und in der Forschungsliteratur zu Fleck schon mehrfach betont wurde), bietet Flecks Denkstillehre ein dynamisches Modell, wonach in Denkkollektiven zwar einerseits homogenisierende Dynamiken am Werk sind und die in ihnen herrschende kollektive Stimmung wesentlich auf Angleichung hinausläuft. Andererseits aber geht Fleck von einer grundlegenden Offenheit von Denkkollektiven aus. In der hier untersuchten Konstellation zeichnen sich damit mehrere entscheidende Färbungswechsel ab, die Fleck am Verhältnis zwischen Individuum und Kollektiv vorgenommen und auf die grundsätzliche Problematik der kollektiven Erkenntnisgewinnung appliziert hat. Was die Fleck'sche Denkstillehre ausmacht, sind immer wieder zwei Charakteristika: Zum einen die unbedingte Parteinahme für Dynamik und gegen Statik, für Offenheit statt Geschlossenheit, für das (bewegliche) Kollektiv und gegen das autonome Individuum. Zum zweiten und eng damit verbunden: die Suche nach Mechanismen zur Entstehung wie auch zur Korrektur von Irrtümern im Kollektiv selbst. Anstatt wie Gumplowicz und Bilikiewicz auf je eigene Weise das autonome Individuum als Bollwerk gegen die fatalen Wirkungen eines „Herdentriebs“, einer Reproduktion und Verstärkung von Irrtümern 
einzusetzen, setzt die Fleck'sche Wissenssoziologie in beiden Aspekten konsequent auf die Dynamik von Kollektiven.

Verglichen mit den beiden Umweltkonzepten von Gumplowicz und Bilikiewicz - hier das individuelle Denken determinierend, dort das individuelle Denken ,verunreinigend' - ging es Fleck offenbar um die Etablierung eines dritten Wegs: das Individuum ist zwar kein autonomes Subjekt, es ist aber auch kein bloßer Reflektor. Gerade weil jedes Individuum die „Stimmung“ eines Kollektivs verändern kann, können durch seinen Einfluss auch stets neue, andere Kollektivgebilde entstehen. Weil er Denkkollektive als atmosphärisch entstehende Gebilde begreift, kann Fleck sie also sowohl von ihrer Offenheit als auch von ihrer Geschlossenheit aus betrachten.

\section{Anmerkungen}

Für gemeinsame Diskussionen und hilfreiche Hinweise danke ich herzlich Nicolas Berg, sowie drei anonymen Gutachter/innen.

1 Inwiefern Fleck dem Unterfangen Jerusalems damit gerecht wird, sei an dieser Stelle dahingestellt. Vgl. zur näheren Diskussion der beiden Ansätze den Beitrag von Julian Bauer in diesem Heft.

2 In zwei Rezensionen in der Klinischen Wochenschrift (Petersen 1936) und der Zeitschrift für Psychologie (Kroh 1936) war Flecks Monografie über die Entstehung und Entwicklung einer wissenschaftlichen Tatsache lobend besprochen und zum Teil als kompatibel mit nationalsozialistischen Anschauungen befunden worden war; ein Manko sah der Rezensent Oswald Kroh allerdings darin, dass Fleck die "Rasse“ als denkstilbildenden Faktor vernachlässigt habe (Kroh 1936: 1).

3 Ludwig Gumplowicz wurde 1838 in Krakau als Sohn eines jüdischen Rabbiners geboren. Nach einem Jurastudium in Krakau war er dort als Anwalt tätig und wurde gleichzeitig in der national-polnischen Widerstandsbewegung aktiv. Aufgrund seiner nationalistischen Agitation und Teilnahme am Januaraufstand der Polen gegen Russland 1863 musste Gumplowicz Krakau verlassen und ging nach Graz, wo er 1875 zum Privatdozenten für Verwaltungslehre und schließlich 1882 zum Außerordentlichen, 1893 zum Ordentlichen Professor ernannt wurde. Das Scheitern der Nationalbewegung sowie das Anschwellen der antijüdischen Pogromwellen ab den 1880er Jahren stürzten Gumplowicz in eine tiefe Resignation, die ihren Niederschlag auch und gerade in einem extrem pessimistisch eingefärbten Konzept von Assimilation fand. 1909 nahm er sich nach Diagnose einer Krebserkrankung zusammen mit seiner Frau das Leben. Eine soziologiegeschichtliche Einordnung nimmt die ausführliche Einleitung zu einer Auswahlsammlung seiner Werke (Brix 1986) vor. In deutscher Sprache sind zur Soziologie Gumplowiczs bislang nur zwei kürzere Dissertationen (Goetze 1969, Boßdorf 2003) sowie ein schmaler Ausstellungskatalog (Müller 1988) erschienen. In polnischer Sprache erschien zuletzt eine ausführlich eingeleitete Werkauswahl mit dem Titel Zwa życia Ludwika Gumplowicza. Wybór tekstów [Die zwei Leben des Ludwik Gumplowicz], vgl. Surman/Moselič 2010.

4 Diesen Aspekt beleuchtet der Aufsatz von Philipp Lenhard (Lenhard 2012). Der Autor geht darin besonders auf die paradoxe Rolle ein, die dem Judentum in Gumplowiczs Theorie des "Rassenkampfs“ zukommt. Aufgrund der ihr zugesprochenen "Passivität“ steht diese "Rasse“ in einem augenscheinlichen Widerspruch zu den Aggressions- und Vereinnahmungstrieben, die Gumplowicz zu allgemeinen Naturgesetzen des Rassenkampfs erhebt. Die führte ihn sogar dazu, dem Judentum eine Verletzung dieser Naturgesetze anzulasten. Den antisemitischen Zug seiner Rassensoziologie deutet Lenhard als eine „groß angelegte Bewältigungsstrategie“, ja als „Versuch, das nicht 
verstehbare - die ewige Verfolgung der Juden, die er am eigenen Leib erfahren hatte - mit wissenschaftlichen Mitteln zu rationalisieren“ (Lenhard 2012: 115). Keinesfalls aber sei Gumplowicz, wie etwa Lukacz es tat, als Vorläufer der NS-Rassentheorie zu betrachten.

5 Tarde wird von Fleck, soweit ich sehe, gar nicht, und von Gumplowicz nur sehr beiläufig erwähnt. Seine Bücher seien Ansammlungen von soziologischen Anregungen, wenn auch keine Soziologie.

6 „Und kann man sich einen Begriff machen, was alles sich hier im Geiste dieses neuen Individiuums zusammengefunden, was sich hier als Niederschlag des geistigen Lebens längst vergangener Generationen in dem Hirn des einen Individuums kondensiert hat? Jahrtausendealte Erfahrungen, die längst in fertigen Anschauungen und Vorstellungen sich auf Generationenreihen vererbten; vorhistorische und historische Schicksale mit ihren geistigen Resultaten in Charakter und Neigungen, mit ihren Gedankenformen und Denkungsarten, jahrtausendealte Sympathien, Vorurteile und Eingenommenheiten, alles das sitzt tief im Geiste des ,freien' Individuums, konzentriert sich in ihm wie Millionen Strahlen in einem Brennpunkt, alles das lebt in ihm als Gedanke, von dem der Haufe wähnt, das Individuum denke ihn in seiner Freiheit; alles das lebt in seinem Gemüte als Gefühl, von dem der Haufe wähnt, das Individuum hege es, mit Recht oder Unrecht, als sein Verdienst oder seine Schuld.“ (Gumplowicz 1905: 270)

7 Der Text des Vortrags nebst dem dazugehörigen Kommentar von Ludwik Hirszfeld ist abgedruckt in Werner/Zittel 2011: 364-368.

8 In der ohnehin schmalen Forschungsliteratur zu Bilikiewicz wurde bislang nicht der Versuch unternommen, seinen Ansatz sowohl in der psychologischen als auch innerhalb der wissenschaftshistorisch-epistemologischen Wissenschaftskultur seiner Zeit zu verorten.

\section{Literatur}

Anonym [Durkheim, Émile], 1885. Dr. Ludwig Gumplowicz - Grundriss der Sociologie (Esquisse d'une sociologie). Revue Philosophique, 20, 627-640.

Berg, Nicolas, 2011. Denkstil. In: Dan Diner, Hg., Enzyklopädie jüdischer Geschichte und Kultur. Bd. 2. Stuttgart: J.B. Metzler, 94-100.

Bilikiewicz, Tadeusz, 1932. Die Embryologie im Zeitalter des Barock und des Rokoko. Leipzig: Thieme.

Bilikiewicz, Tadeusz, 1939a. Bemerkungen zum Artikel von Ludwik Fleck „Wissenschaft und Umwelt". In: Sylwia Werner und Claus Zittel, Hg., 2011. Ludwig Fleck. Denkstile und Tatsachen. Gesammelte Schriften und Zeugnisse. Berlin: Suhrkamp, 340-352.

Bilikiewicz, Tadeusz, 1939b. Antwort auf die Replik von Ludwik Fleck. In: Sylwia Werner und Claus Zittel, Hg., 2011. Ludwig Fleck. Denkstile und Tatsachen. Gesammelte Schriften und Zeugnisse. Berlin: Suhrkamp, 361-363.

Boßdorf, Peter, 2003. Ludwig Gumplowicz als materialistischer Staatssoziologe. Eine Untersuchung zur Ideengeschichte der Soziologie. Diss. Universität Bonn.

Borck, Cornelius, 2004. Message in a Bottle From „the Crisis of Reality”: On Ludwik Fleck's Interventions for an Open Epistemology. Studies in the History of Biology and Biomedical Sciences, 35, 447-464.

Brix, Ludwig, Hg., 1986. Gumplowicz oder die Gesellschaft als Natur. Wien: Böhlau.

Durkheim, Emile/Mauss, Marcel, 1901/1902. De quelques formes primitives de classification. Année sociologique, 6, 1-72.

Fagan, Melinda B., 2009. Fleck and the Social Constitution of Scientific Objectivity. Studies in History and Philosophy of Biological and Biomedical Sciences, 40, 272-285.

Fleck, Ludwik, 1935. Entstehung und Entwicklung einer wissenschaftlichen Tatsache. Einführung in die Lehre vom Denkstil und Denkkollektiv. Frankfurt a. M.: Suhrkamp 1980.

Fleck, Ludwik, 1939a. Wissenschaft und Umwelt. In: Sylwia Werner und Claus Zittel, Hg., 2011. Ludwig Fleck. Denkstile und Tatsachen. Gesammelte Schriften und Zeugnisse. Berlin: Suhrkamp, 327-339. 
Fleck, Ludwik, 1939b. Antwort auf die Bemerkungen von Tadeusz Bilikiewicz. In: Sylwia Werner und Claus Zittel, Hg., 2011. Ludwig Fleck. Denkstile und Tatsachen. Gesammelte Schriften und Zeugnisse. Berlin: Suhrkamp, 353-368.

Fleck, Ludwik, 1946. Wissenschaftstheoretische Probleme. In: Sylwia Werner und Claus Zittel, Hg., 2011. Ludwig Fleck. Denkstile und Tatsachen. Gesammelte Schriften und Zeugnisse. Berlin: Suhrkamp, 369-389.

Fleck, Ludwik, 1947. Schauen, Sehen, Wissen. In: Sylwia Werner und Claus Zittel, Hg., 2011. Ludwig Fleck. Denkstile und Tatsachen. Gesammelte Schriften und Zeugnisse. Berlin: Suhrkamp, 390-418.

Fleck, Ludwik, 1960. Krise in der Wissenschaft. Zu einer freien und menschlichen Wissenschaft. In: Sylwia Werner und Claus Zittel, Hg., 2011. Ludwig Fleck. Denkstile und Tatsachen. Gesammelte Schriften und Zeugnisse. Berlin: Suhrkamp, 466-474.

Gieryn, Thomas, 1983. Boundary-work and the Demarcation of Science from Non-science: Strains and Interests in Professional Ideologies of Scientists. American Sociological Review, 48, 781795.

Goetze, Dieter, 1969. Die Staatstheorie von Ludwig Gumplowicz oder die Gesellschaft als Natur. Diss. Univ. Heidelberg.

Gumplowicz, Ludwig, 1883. Der Rassenkampf. Soziologische Untersuchungen. Leipzig: Wagner.

Gumplowicz, Ludwig, 1899. Die Wechselbeziehungen der sozialen Gebilde. Die Zukunft, 26, 366371.

Gumplowicz, Ludwig, 1905. Grundriss der Soziologie. 2. Aufl. [OA 1885], Wien: Manzsche k. u. k. Hof-Verlags- und Universitäts-Buchhandlung.

Horowitz, Irving Louis, 1980. Introduction. In: Ders., Hg., Ludwig Gumplowicz, Outlines of Sociology [Grundriss der Soziologie, OA 1885]. New Brunswick/NJ: Paine-Whitman Publishers, 10-85.

Jerusalem, Wilhelm, 1924. Die soziale Bedingtheit des Denkens und der Denkformen. In: Meja, Volker und Nico Stehr Hg., 1982. Der Streit um die Wissenssoziologie. Bd. 1: Die Entwicklung der deutschen Wissenssoziologie. Frankfurt a. M.: Suhrkamp, 27-56.

Kroh, Oswald, 1936. Rezension von Ludwik Flecks „Entstehung und Entwicklung einer wissenschaftlichen Tatsache". Zeitschrift für Psychologie, 137, 1-3.

Lenhard, Philipp, 2012. Assimilation als Untergang. Ludwig Gumplowicz' Judentum und die Frage des Antisemitismus. Zeitschrift für Religions- und Geistesgeschichte, 64/2, 105-116.

Löwy, Ilana, 1990. Ludwig Fleck. From Philosophy of Medicine to an Constructivist and Relativist Epistemology. In: dies., The Polish School of Philosophy of Medicine. Dordrecht: Kluwer, 215227.

Müller, Reinhard, Hg., 1988. Ludwig Gumplowicz (1838-1909). Ein Klassiker der Soziologie. Katalog zur Ausstellung an der Universitätsbibliothek Graz anlässlich des 150. Geburtstages von Ludwig Gumplowicz. Graz: Universitätsbibliothek.

Petersen, Hans, 1936. Ludwig Fleck's Lehre vom Denkstil und dem Denkkollektiv. Klinische Wochenschrift, 15, 239-242.

Surman, Jan/Moselič, Gerard, Hg., 2010. Zwa życia Ludwika Gumplowicza. Wybór tekstów, Warzaw: Oficyna Naukowa.

Tarde, Gabriel, 2009 [OA 1890]. Die Gesetze der Nachahmung, Frankfurt a. M.: Suhrkamp.

Werner, Sylwia/Zittel, Claus, Hg., 2011. Ludwig Fleck. Denkstile und Tatsachen. Gesammelte Schriften und Zeugnisse. Berlin: Suhrkamp 2011.

Werner, Sylwia, 2011. Wissenschaft und Magie. Ethnologische Motive in Ludwik Flecks Methodologie. Vortrag im Forschungskolloquium an der Professur für Wissenschaftsforschung an der ETH Zürich, 21. November 2011.

Zittel, Claus, 2007. Die Entstehung und Entwicklung von Ludwik Flecks vergleichender „Erkenntnistheorie”. In: Bozena Chołuj und Jan C. Joerden, Hg., Von der wissenschaftlichen Tatsache zur Wissensproduktion: Ludwik Fleck und seine Bedeutung für die Wissenschaft und Praxis. Frankfurt a. M.: Peter Lang, 439-466.

Zittel, Claus, 2011. Ludwik Fleck und der Stilbegriff in den Naturwissenschaften. Stil als wissenschaftshistorische, epistemologische und ästhetische Kategorie. In: Horst Bredekamp und John Michael Krois, Hg., Sehen und Handeln (= Actus et Imago. Berliner Schriften für Bildaktforschung, Bd. 1). Berlin: Akademie-Verlag, 171-206. 
Eva Johach

Kulturwissenschaftliches Kolleg

Universität Konstanz

Otto-Adam-Str. 5

78467 Konstanz

Germany

E-Mail: eva.johach@uni-konstanz.de 